\title{
Quantum Zeno effect for a free-moving particle
}

\author{
Miguel A. Porras \\ Grupo de Sistemas Complejos, Universidad Politécnica de Madrid, Rios Rosas 21, 28003 Madrid, Spain
}

Alfredo Luis and Isabel Gonzalo

Departamento de Óptica, Facultad de Ciencias Físicas, Universidad Complutense, 28040 Madrid, Spain

(Received 13 October 2014; published 29 December 2014)

\begin{abstract}
Although the quantum Zeno effect takes its name from Zeno's arrow paradox, the effect of frequently observing the position of a freely moving particle on its motion has not been analyzed in detail in the frame of standard quantum mechanics. We study the evolution of a moving free particle while monitoring whether it lingers in a given region of space, and explain the dependence of the lingering probability on the frequency of the measurements and the initial momentum of the particle. Stopping the particle entails the emergence of Schrödinger cat states during the observed evolution, closely connected to the high-order diffraction modes in Fabry-Pérot optical resonators.
\end{abstract}

DOI: 10.1103/PhysRevA.90.062131

PACS number(s): 03.65.Xp, 03.65.Ta, 42.25.Fx, 42.60.Da

\section{INTRODUCTION}

According to Zeno's approach to dynamics, flying arrows are always at rest because at any instant they are at some position, which is interpreted in Zeno's paradox as being at rest. The conclusion turned out to be false, since being instantaneously anywhere is actually compatible with motion, as demonstrated by infinitesimal calculus. Despite this failure, quantum mechanics offered a revival to Zeno's paradox when asking what happens when we actually monitor where the arrow is. It emerged that the observation alters the evolution, even to the extent of stopping it $[1,2]$.

The first approaches to the observed evolution focused on monitoring the state of the system, not on observing the position of a traveling object like the flying arrow, an issue that then deserved little attention [3]. In this work we step into the arrow's flight by observing whether a freely moving object is located within a given spatial region (every position observation must be on a finite region). This problem falls within the context of the so-called quantum Zeno dynamics, to distinguish it from the earlier Zeno effect [4]. The standard Zeno effect deals with the observation of the system state. Zeno dynamics deals with the observation of a dynamical property, the spatial location in our case, while the system state is otherwise free to evolve.

The system preparation and observation are presented in Sec. II, where Zeno's arrow is represented by a onedimensional particle with some initial linear momentum $p$ evolving freely. In a computational approach, we obtain in Sec. III the probability that the particle remains in the location where it is initially found, recovering previous results of unit lingering probability in the limit of continuous observation [4]. Below this ideal limit, nevertheless, our analysis reveals that it is harder to stop the particle (it needs more frequent measurements) as the particle has an initial large momentum with negligible uncertainty $(\Delta p / p \rightarrow 0)$, like the classical Zeno's arrow. In Sec. IV, we explain the numerical results in terms of the emergence during the observed evolution of Schrödinger cat states as the coherent superposition of two arrows traveling forward and backward (a Schrödinger arrow state, so to speak). Thus, below the limit of continuous measurement, the particle is not only trapped in the subspace defined by the measurement, but is actually stopped in a highly nonclassical state. The structure of this Schrödinger cat state corresponds to what can be called a high-order time-diffraction mode - a well-known wave object in the theory of open optical resonators in wave optics [5,6]. Diffraction concepts in optics are adapted to time diffraction in the quantum context [7] to obtain a simple expression for the lingering probability as a function of the frequency of the measurements that reproduces the features described numerically.

In comparison with related previous works on quantum Zeno dynamics, such as Refs. [3] and [8], the system that we analyze is extremely simple and close to the original Zeno paradox, we reveal and interpret finer details of the Zeno dynamics below the limit of continuous measurements, drawing many parallels with similar problems in classical wave optics. This example provides moreover a suitable arena for simple experimental implementations of Zeno dynamics, as already demonstrated in previous works [9].

Similar schemes have been proposed as a natural way to determine the distribution of arrival times, or the probability that a particle spends a certain time period in a given region. These two problems have been interwoven from the very beginning of the modern history of the Zeno effect [10]. Nevertheless, in practical terms they tend to diverge since in the Zeno effect the standard formalism is used to compute probabilities at given time instants. Useful details about the difficulties and efforts of formalizing time in quantum mechanics may be found in Ref. [11].

\section{AN OBSERVED FREELY MOVING PARTICLE}

For simplicity, we consider a free particle of mass $m$ moving in one dimension $x$. At a time $t=0$, the wave function of the particle is described by $\psi_{T}(x, 0)=\psi(x) \exp (i p x / \hbar) \operatorname{rect}(x / a)$, where $\operatorname{rect}(\xi)=1$ for $|\xi| \leqslant 1$ and 0 otherwise, with

$$
\int_{-a}^{a} d x|\psi(x)|^{2}=1 .
$$

The particle is then confined in $[-a, a]$ with a probability density $|\psi(x)|^{2}$, and moves at a velocity $v=p / m$. As time 
passes, a measurement device is used to monitor whether the particle remains in $[-a, a]$ or not. In the negative case, a click is heard and the particle is eliminated from the system. After a time $t$, the probability that the particle remains in $[-a, a]$ is

$$
P_{1}=\int_{-a}^{a} d x|\psi(x, t)|^{2},
$$

where $\psi(x, t)$ is the wave function at the time $t$ given by the time-dependent Schrödinger equation

$$
i \hbar \frac{\partial \psi}{\partial t}=-\frac{\hbar^{2}}{2 m} \frac{\partial^{2} \psi}{\partial x^{2}}
$$

with the initial condition $\psi_{T}(x, 0)$. After a positive measurement, the particle is certainly in $[-a, a]$ so that the state of the particle is described by the wave function $\psi_{T}(x, t)=\psi(x, t) \operatorname{rect}(x / a) / \sqrt{P_{1}}$, i.e., is projected in $[-a, a]$ and normalized to unity. The probability $P_{1}$ is smaller than unity because the wave function translates on average at velocity $v$, and also because it spreads.

Suppose now that we perform $N$ of the above measurements within the time $t$ at equispaced intervals $t / N$. Starting again with $\psi_{T}(x, 0)$, the wave function experiences $N$ of the above cascaded transformations. For example, from the $(n-1)$ th to the $n$th measurement $(n=1,2, \ldots, N)$,

$$
\begin{gathered}
\psi_{T}\left(x, \frac{(n-1) t}{N}\right) \stackrel{\text { evol }}{\rightarrow} \psi\left(x, \frac{n t}{N}\right) \\
\stackrel{\text { meas }}{\rightarrow} \psi_{T}\left(x, \frac{n t}{N}\right)=\psi\left(x, \frac{n t}{N}\right) \frac{\operatorname{rect}(x / a)}{\sqrt{P_{N}^{(n)}}},
\end{gathered}
$$

where

$$
P_{N}^{(n)}=\int_{-a}^{a} d x\left|\psi\left(x, \frac{n t}{N}\right)\right|^{2}
$$

is the probability that the particle is found in $[-a, a]$ at the $n$th measurement after having been found in $[-a, a]$ at the $(n-1)$ th measurement. After the time $t$, the particle is in $[-a, a]$ only if all measurements were positive, with a probability equal to the product of the individual probabilities:

$$
P_{N}=\prod_{n=1}^{N} P_{N}^{(n)} .
$$

The particle can be said to slow down its free motion during the time interval $t$ of frequent observations if the probability $P_{N}$ of finding the particle in the original position $[-a, a]$ is greater than the probability $P_{1}$ with only a final measurement. In this situation, a Zeno effect can be said to occur in the moving free particle.

For more general quantum systems, Facchi and Pascazio [4] have shown that in the limit of infinitely frequent measurements $N \rightarrow \infty$, which is considered to be equivalent to a continuous measurement, the particle becomes trapped in an infinite potential well in $[-a, a]$, experiencing a unitary evolution, or Zeno dynamics, preserving the norm $\left(P_{\infty}=1\right)$. This approach does not consider the nonunitary $\left(P_{N}<1\right)$ Zeno dynamics below this limit, and how it is reached for large and increasing $N$. In particular, trapping the particle is independent of its dynamical properties in the limit of continuous observation, but this is not true with a finite number of measurements.

\section{ZENO EFFECT IN THE OBSERVED FREELY MOVING PARTICLE}

The physical magnitudes that are actually at work in this problem are evidenced by introducing the dimensionless distance $\xi=x / a$ and the dimensionless time $\tau=(v / 2 a) t$, i.e., the time in units of the time $2 a / v$ that the wave function takes to travel its own length $2 a$, so that at $\tau=1$ the probability of finding a classical particle in its original location is zero. With these variables, the initial wave function reads $\psi_{T}(\xi, 0)=\psi(\xi) \exp (i s \xi) \operatorname{rect}(\xi)$, where $s=p a / \hbar=m v a / \hbar$ is an action in units of $\hbar$. Starting with $\psi(\xi)$ such that

$$
\int_{-1}^{1} d \xi|\psi(\xi)|^{2}=1
$$

cascaded free evolution from one measurement to another is performed according to the normalized Schrödinger equation

$$
\frac{\partial \psi}{\partial \tau}=\frac{i}{s} \frac{\partial^{2} \psi}{\partial \xi^{2}},
$$

measurements are performed as in Eq. (5) with $\operatorname{rect}(\xi) / P_{N}^{(n)}$, where

$$
P_{N}^{(n)}=\int_{-1}^{1} d \xi\left|\psi\left(\xi, \frac{n \tau}{N}\right)\right|^{2},
$$

and the probability of finding the particle in its original location $[-1,1]$ after the $N$ measurements is $P_{N}=\prod_{n=1}^{N} P_{N}^{(n)}$. The natural time at which to enquire whether the particle remains in $[-1,1]$ is the time $\tau=1$ that the particle would classically take to leave $[-1,1]$. Once this time is fixed, the dynamics of the observed free particle depends only on the number of measurements $N$ and on the dimensionless action $s$.

We are particularly interested in the behavior of $P_{N}$ as $N$ is large, and in particles featuring a definite translation movement, like Zeno's arrow. In the quantum context this means that the wave function does not experience a strong deformation due to time diffraction spreading at $\tau=1$. Imposing then the requirement that the characteristic time $m a^{2} / \hbar$ of time diffraction is longer than $2 a / v$, we find the condition $s>2$ for the action. The Zeno dynamics in the opposite regime where time-diffraction spreading dominates over translation $(s<2)$ has been studied in Ref. [9]. In the limit $s \rightarrow \infty$, the freely evolving wave function does not experience any transformation other than a rigid translation with a fully defined momentum, which can be considered in this context to represent the limit of a moving classical particle. Indeed, for wave packets obeying the Heisenberg uncertainty relation $a \Delta p \sim \hbar$, we have $\Delta p / p \sim 1 / s$, meaning that the uncertainty $\Delta p$ in momentum becomes negligibly small compared to $p$ in the limit $s \rightarrow \infty$.

We have simulated with a numerical code the problem described above in a grid of points $\xi_{i}$ of finite size, verifying in all simulations that the grid size and number of points are large enough so that any effects of finiteness and discretization are negligible. The particular choice of the initial, smooth wave function $\psi(\xi)$ filling the region $[-1,1]$ does not alter 

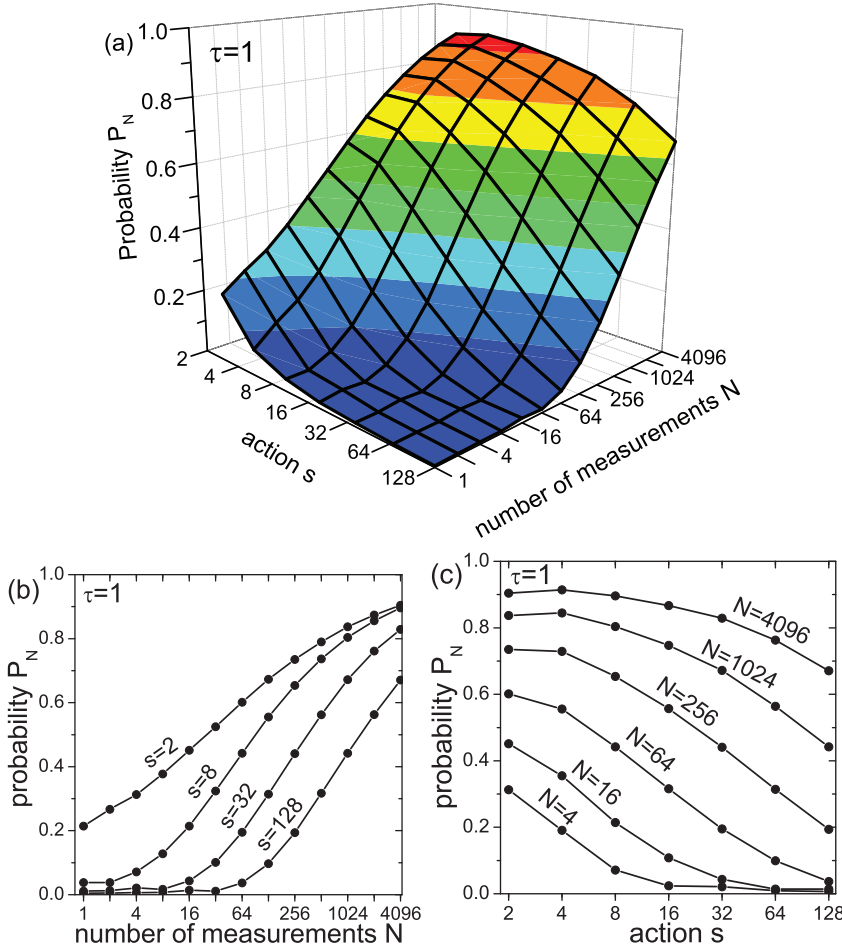

FIG. 1. (Color online) (a) Probability $P_{N}$ that a moving particle remains in its original location at the time that the particle would classically take to leave it, as a function of the number $N$ of intermediate measurements and the action $s$ of the particle. (b) Probability on increasing the number of measurements and fixed action. (c) Probability on increasing the action and fixed number of measurements.

substantially the results described below, particularly for large $N$. For convenience we have chosen the Gaussian wave function $\psi(\xi) \propto \exp \left(-\xi^{2}\right)$ in most of the numerical simulations.

Figure 1(a) shows the probability $P_{N}$ that the particle is found in $[-1,1]$ at $\tau=1$ for different values of $N$ and $s$. The probability $P_{N}$ for fixed values of $s$ and variable $N$ and for fixed $N$ and variable $s$ are displayed in Figs. 1(b) and 1(c), respectively. As seen, $P_{N}$ always increases with the number of measurements $N$, approaching unity in the limit $N \rightarrow \infty$, as expected from [4] and regardless of the action $s$ of the particle [Fig. 1(b)]. However, as $s$ increases, more and more measurements are needed to obtain the same increase of the probability. Indeed, the probability of stopping the particle with any given value of measurements $N$ approaches zero as the action of the particle increases [Fig. 1(c)]. This means that the Zeno effect is less effective as the particle becomes classical, and disappears as $s \rightarrow \infty$. Provided $N$ is large, the probability $P_{N}$ is numerically found to depend only on the quotient $N / s$. Thus, as the action $s$ is increased by a certain factor, the number of measurements must be increased by the same factor in order to maintain the probability.

As a more specific example, Fig. 2(a) shows the squared modulus of the wave function of a particle with $s=8$ at the initial time $\tau=0$ (dashed curve) and at the final time $\tau=1$ after $N=4,16,128$, and 1024 measurements (solid curves). For a visualization of the increasing probabilities
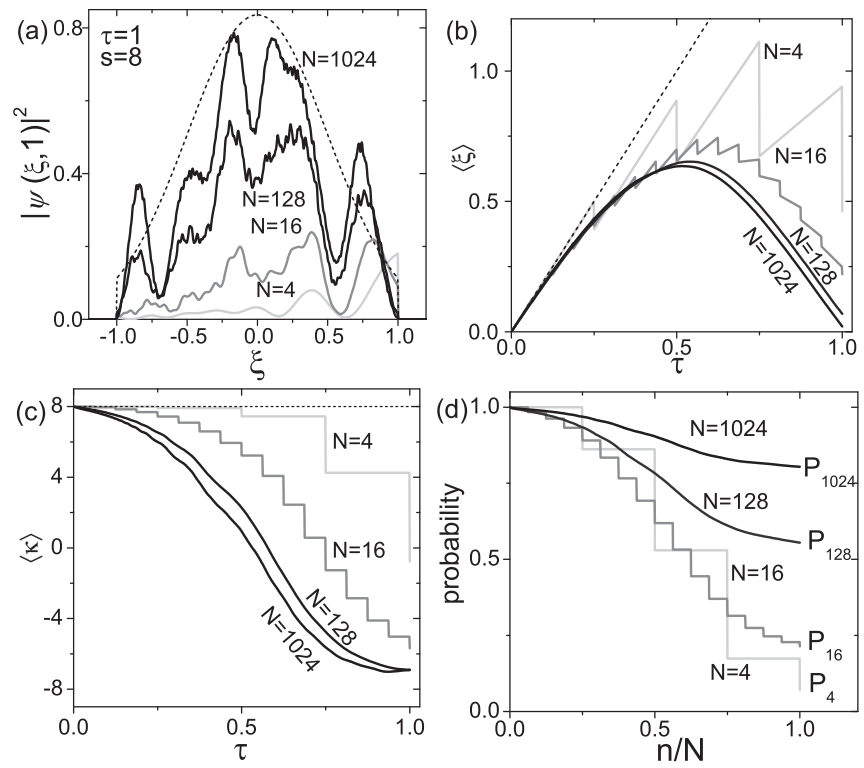

FIG. 2. For a particle with $s=8$ initially in $[-1,1]$, (a) probability density before (dashed curve) and after free evolution for a time $\tau=1$ with $N$ intermediate measurements; (b), (c) time evolution of the expected values of the position $\langle\xi\rangle$ and momentum $\langle\kappa\rangle$ during free evolution with $N$ intermediate measurements; (d) probability that the particle remains in $[-1,1]$ at the $n$th intermediate measurement as a function of the time $\tau=n / N$ of the measurement.

$P_{N}$, the wave function is not normalized to unity after the last measurement, but to $P_{N}$. Some aspects of the Zeno dynamics from $\tau=0$ to $\tau=1$ can be seen in Figs. 2(b)-2(d). Figures 2(b) and 2(c) depict the temporal evolution of the expected values of the position $\langle\xi\rangle$ and of the momentum $\langle\kappa\rangle$ $\left[\kappa=-i \partial_{\xi}=-i(a / \hbar) \partial_{x}\right.$ is the dimensionless momentum] in the case that the particle is found in $[-1,1]$ at $\tau=1$. The values for the unobserved motion are indicated by dashed lines. Each measurement causes a sudden shift in the mean position and momentum, resulting in an almost reflected motion at $\tau=1$. Except for a very small number of measurements, this effect is seen to be substantially independent of $N$ and $s$. To illustrate how the increase of the final probability at $\tau=1$ is furnished during the observed motion, Fig. 2(d) shows the probability $\prod_{i=1}^{n} P_{N}^{(i)}$ that the particle remains in $[-1,1]$ after the $n$th intermediate measurement as a function of time $\tau=n / N$. While in the limit of infinite measurements this probability is always unity, with finite $N$ this probability diminishes in each intermediate measurement. The point to be understood is why an increasing number of measurements, each one causing a diminution of the probability, i.e., increasing the number of steps down in Fig. 2(d), leads to a higher final probability $P_{N}<1$ at $\tau=1$, and why this probability depends on the action of the particle.

\section{HIGH-ORDER TIME-DIFFRACTION MODES IN THE ZENO DYNAMICS OF THE OBSERVED FREELY MOVING PARTICLE}

An explanation of the above facts requires a closer look at the dynamics of the observed free evolution. If we prolong it up to longer times (maintaining the frequency of the 

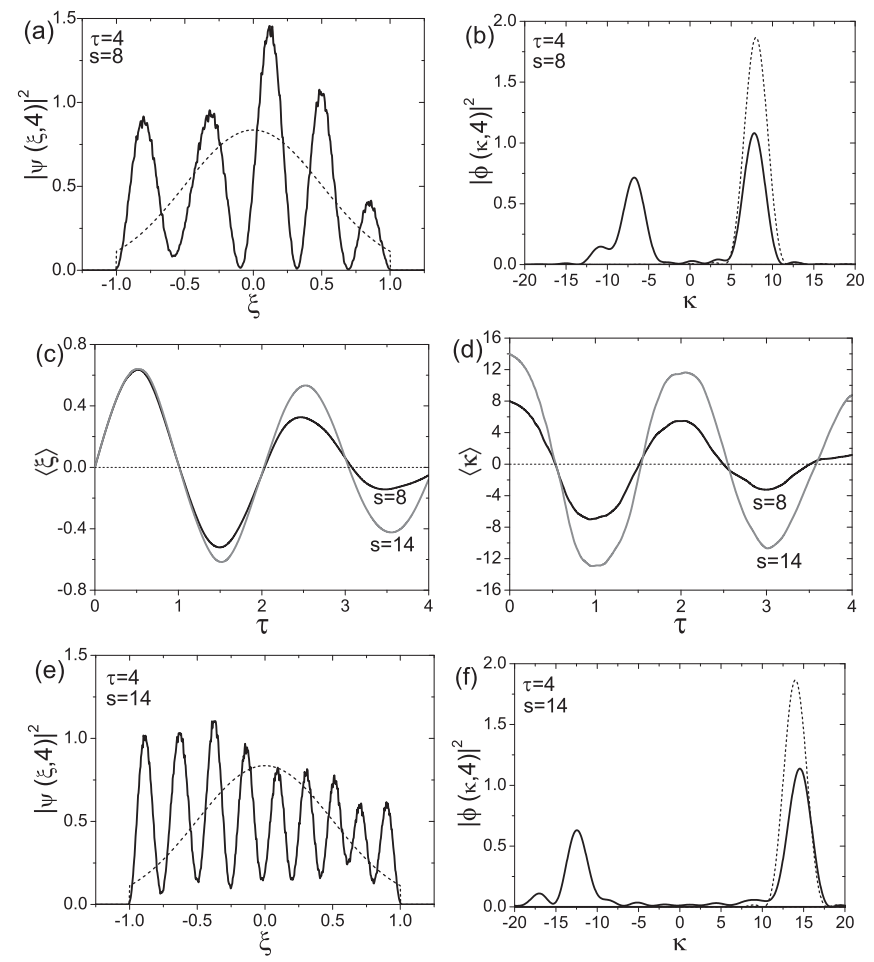

FIG. 3. (a), (b) For a particle with $s=8$ initially in $[-1,1]$, the position probability densities and momentum probability densities before (dashed curves) and after free evolution for a time $\tau=4$ with $N=4096$ intermediate measurements (solid curves). (c), (d) For particles with $s=8$ and $s=14$ initially in $[-1,1]$, the time evolution of the expected values of the position $\langle\xi\rangle$ and momentum $\langle\kappa\rangle$ during free evolution with $N=4096$ intermediate measurements. (e), (f) The same as in (a) and (b) except that $s=14$.

measurements), e.g., from $\tau=1$ with $N=1024$ to $\tau=4$ with $N=4096$ in Fig. 3, we observe that the incipient wave structure with five main maxima (for $s=8$ ) already observed at $\tau=1$ [Fig. 2(a)] becomes more sharply defined with (close to) zero intermediate minima [Fig. 3(a)]. In momentum representation, the wave function

$$
\phi(\kappa, \tau)=\frac{1}{\sqrt{2 \pi}} \int d \xi \psi(\xi, \tau) \exp (-i \xi \kappa),
$$

with an initial single probability maximum at $\kappa=s$, retains that maximum at $\kappa \simeq s$ and develops a symmetric maximum at $\kappa \simeq-s$ [Fig. 3(b)]. The relative amplitude of the different maxima in $|\psi(x, \tau)|^{2}$ fluctuate with time, making the average position $\langle\xi\rangle$ also oscillate in time, and the relative amplitudes of the two maxima of $|\phi(\kappa, \tau)|^{2}$ also fluctuate, making the average momentum $\langle\kappa\rangle$ oscillate also. All these fluctuations are however damped in time, as observed in Figs. 3(c) and 3(d) for the average position and momentum. This description holds irrespective of the value of $s>2$ provided that the number of measurements $N$ is large, except the number of maxima formed in the long-term evolution. In Figs. 3(c)-3(f) for $s=$ 14 , for example, the same behavior is observed except that the number of maxima in $|\psi(\xi, \tau)|^{2}$ is nine and the two maxima of $|\phi(\kappa, \tau)|^{2}$ are centered at $\kappa \simeq \pm 14$.

The dynamics above strongly recalls the well-known optical-wave dynamics of the formation of high-order diffrac- tion modes in a Fabry-Pérot resonator (or equivalently, the high-order diffraction modes in cascaded diffracting apertures). Translated to the quantum mechanical language and notation, a "time-diffraction" mode is a solution $\psi_{l}(\xi)$ of the integral equation

$$
\int_{-a}^{a} d x^{\prime} K\left(x^{\prime}-x\right) \psi_{l}\left(x^{\prime}\right)=\gamma_{l} \psi_{l}(x)
$$

where

$$
K\left(x^{\prime}-x\right)=\left(\frac{m}{2 \pi i \hbar \Delta t}\right)^{1 / 2} \exp \left[i \frac{m}{2 \hbar \Delta t}\left(x^{\prime}-x\right)^{2}\right]
$$

is the kernel of the free-particle propagator for a time $\Delta t$ between two measurements. Equation (12) states that a mode $\psi_{l}(x)$ self-reproduces in $[-a, a]$ from one projective measurement to a subsequent measurement after a time $\Delta t$ (from one to another planar mirror of width $2 a$ separated by a certain distance), aside from a complex constant $\gamma_{l}$. Given $\Delta t$ and the interval $[-a, a]$, there exists an infinite numerable set of modes $\psi_{l}(\xi), l=1,2, \ldots$, whose probability distributions $\left|\psi_{l}(\xi)\right|^{2}$ have $l$ maxima and $l-1$ intermediate nodes. In this respect, these time-diffraction modes resemble the stationary states of an infinite potential well in $[-a, a]$. In particular, for high-order $l$, the $l$ maxima in $2 a$ of the mode $\psi_{l}(\xi)$ imply that the wave function $\phi_{l}(p)$ in momentum representation features two opposite maxima at $p \simeq \pm \hbar \pi l / 2 a$. They differ from perfect standing waves in that they are not zero at $\pm a$. A portion of the wave function escapes from $[-a, a]$ during the time $\Delta t$ and is therefore removed in the projective measurement, which causes the probability of finding the particle in $[-a, a]$ (the light power in optics) after the measurement to be reduced by a factor $\left|\gamma_{l}\right|^{2}$. This quantity is well known in the context of optical resonators, and is given, in quantum mechanical notation, by [6]

$$
\left|\gamma_{l}\right|^{2} \simeq 1-0.12 l^{2}\left(\frac{2 \pi \hbar \Delta t}{m a^{2}}\right)^{3 / 2},
$$

provided that $\left(m a^{2} / 2 \pi \hbar \Delta t\right)$ (the resonator Fresnel number) is larger than unity. Evaluation of the exact shape of the diffraction modes requires solving the integral equation (12). Alternatively, and in a more practical approach, a wave mode $\psi_{l}(x)$ defined by Eq. (12) tends to emerge spontaneously upon repeated projections in $[-a, a]$ at time intervals $\Delta t$ (repeated bounces in the resonator) starting from a suitable excitation $\psi_{T}(x, 0)$.

This theory and the above numerical results suggest that a large number $N$ of projective measurements in $[-a, a]$ performed within a time $t$, starting with a particle in $[-a, a]$ with a momentum wave function $\phi_{T}(p, 0)$ sharply peaked at $p(\Delta p / p \sim 1 / s)$, tends to excite the mode in $[-a, a]$ with $\Delta t=t / N$ and the order $l$ such that its momentum maximum $\hbar \pi l / 2 a$ is closest to $p$, that is, the mode

$$
l \approx \frac{2 a p}{\pi \hbar}=\frac{2}{\pi} s
$$

This relation accounts, for example, for the $l=5$ and $l=9$ maxima for excitation with $s=8$ and $s=14$, respectively, in Fig. 3. Equation (14) with $\Delta t=t / N$ and the above value of 

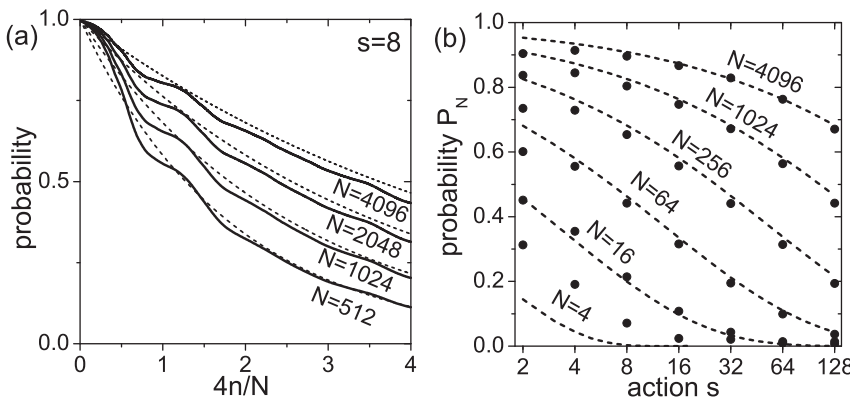

FIG. 4. (a) For $s=8$, and for several numbers of total measurements $N$ within $\tau=4$, the probability that the particle remains in $[-1,1]$ at the $n$th intermediate measurement as a function of the time $\tau=4 n / N$ of the measurement. The solid curves represent the numerically evaluated values and the dashed curves the theoretical predictions. (b) Probability $P_{N}$ that a moving particle remains in $[-1,1]$ after $N$ intermediate measurements within $\tau=1$ as a function of the action $s$ of the particle. The circles represent the numerically evaluated probabilities and the dashed curves the predictions of Eq. (17).

$l$ yields

$$
\begin{aligned}
P_{N}^{(n)} & \approx 1-0.12\left(\frac{2 a p}{\pi \hbar}\right)^{2}\left(\frac{2 \pi \hbar t}{m a^{2} N}\right)^{3 / 2} \\
& =1-0.12\left(\frac{2}{\pi} s\right)^{2}\left(\frac{4 \pi \tau}{N s}\right)^{3 / 2}
\end{aligned}
$$

for the probability of finding the particle in $[-a, a]$ in the $n$th measurement after having been found in $[-a, a]$ in the $(n-1)$ th measurement, under the assumption that the mode is already formed at the times of these two measurements. This probability is independent of $n$, and increases with increasing $N$ [the heights of the steps down in Fig. 2(b) diminish with increasing number of steps, $N$ ] fast enough so that the probability that the particle remains in $[-a, a]$ after the $N$ th measurement at $\tau$, given by

$$
P_{N}=\prod_{n=1}^{N} P_{N}^{(n)} \approx\left\{1-0.12\left(\frac{2}{\pi} s\right)^{2}\left(\frac{4 \pi \tau}{N s}\right)^{3 / 2}\right\}^{N},
$$

approaches unity as $N$ increases. Also, for $N \rightarrow \infty$, use of Newton's binomial theorem leads to the approximate asymptotic expression $P_{N} \approx 1-1.08(s \tau / N)^{1 / 2}$, which accounts for the numerically observed dependence of the probability on the quotient $N / s$ in this limit, and for the increasing difficulty of stopping the particle as its momentum becomes larger, as in the case of classical arrows.

As a theory-numerics comparison, Fig. 4(a) represents the probability $\prod_{i=1}^{(n)} P_{N}^{(i)}$ that the particle remains in $[-1,1]$ after the $n$th intermediate measurement simulated numerically (solid curves) and given by the above theory (dashed curves) for a particle with $s=8$ and several values of the number of total measurements $N$ within $\tau=4$. The good agreement between theory and numerics is surprising if we consider that the higher-order mode is formed only gradually from the initial wave function $\psi_{T}(x, 0)$ (the mode is not even fully formed at the final time $\tau=4$, as seen in Fig. 3).
Finally, the circles in Fig. 4(b) replicate the numerically obtained values of the probability already shown in Fig. 1(c), that is, the probability of finding the particle in $[-1,1]$ at $\tau=1$ as a function of the action $s$ of the particle for a few values of $N$. The dashed curves in Fig. 4(b) represent the theoretical predictions of Eq. (17). These curves are seen to explain the Zeno effect of freezing the particle as the number of measurements increases, and its gradual ineffectiveness as the action of the particle increases.

\section{CONCLUSIONS}

We have analyzed a simple implementation of the observation of the free evolution of a system mimicking the famous problem of Zeno's arrow. We have shown that a moving object tends to be stopped by observation of its location with a probability that always increases with the frequency of the observations. For a given observation rate, on the other side, the effectiveness of stopping the object decreases as the momentum and size of the object increases, which brings to mind the fatuousness of stopping a classical object like Zeno's arrow by simple observation.

The wave function of the stopped particle tends to be similar to a high-order diffraction mode of a Fabry-Pérot resonator, with a symmetric momentum distribution about $p=0$ and therefore $\langle p\rangle=0$. Measurements introduce lossy reflections that create a coherent superposition of the initial system with its equivalent moving backwards, leading to a neat example of a Schrödinger cat state. Since the system state is always pure, a momentum distribution such as in Fig. 3 reveals the coherent superposition of two arrows flying in opposite directions.

We emphasize that mode formation and the emergence of the Schrödinger cat states hold just in the case of finite $N$.

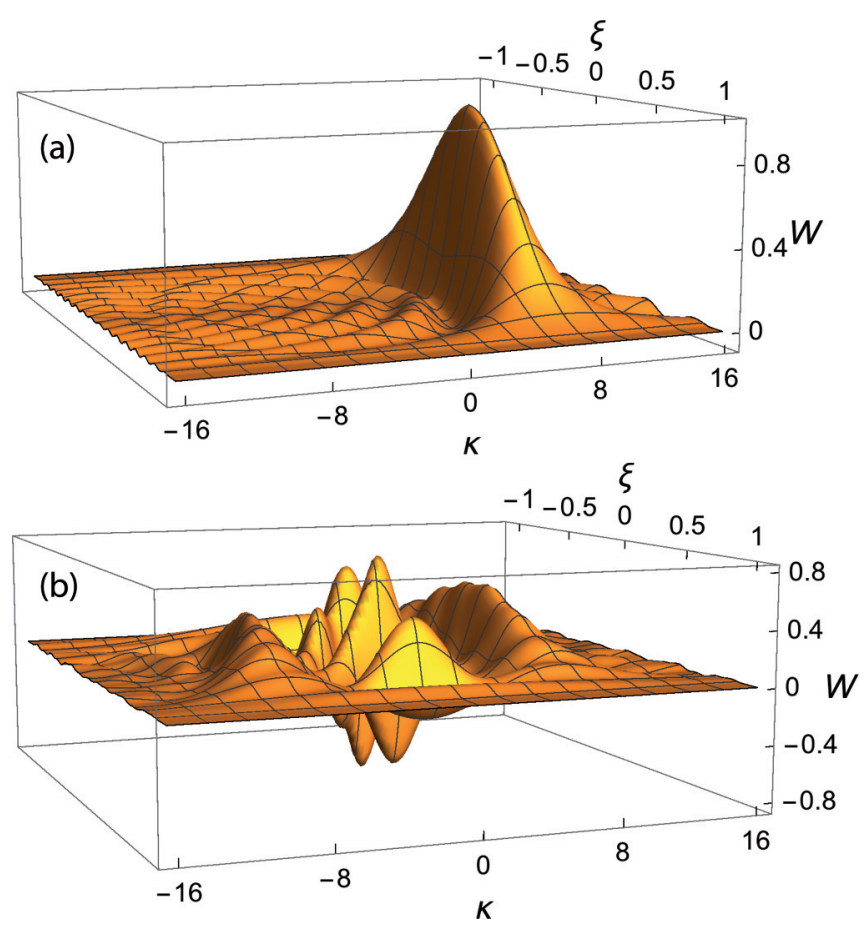

FIG. 5. (Color online) Wigner functions for (a) the initial and (b) evolved states in Figs. 3(a) and 3(b). 
Otherwise, in the infinite limit, we will get a particle bouncing back and forth within the interval, so that the two opposite momentum values will not coexist. No stationary distribution will be approached. The strange nature of such Schrödinger cat state can be well expressed by the negativities of its Wigner function [12],

$$
W(\xi, \kappa)=\frac{1}{\pi} \int d u \psi(\xi+u) \psi^{*}(\xi-u) e^{-2 i \kappa u},
$$

which is considered to be the best candidate for a simultaneous distribution of position and momentum. In Fig. 5(a) we have represented the Wigner function of the initial state in Figs. 3(a) and $3(\mathrm{~b})$. The Wigner function shows no negativities other than small ripples caused by the truncation of the initial Gaussian wave function. On the other hand, the Wigner function of the evolved state in Fig. 5(b), corresponding to the solid curves in Figs. 3(a) and 3(b), displays clear negativities where the typical structure of a Schrödinger cat can be recognized. There are two large positive-momentum peaks located at $\kappa= \pm 8$ and in between a series of positive and negative interference peaks reflecting the coherent nature of the superposition. These negativities reveal a clear incompatibility of the evolved particle with classical physics. Alternatively, this structure has a natural counterpart in classical wave optics as indicating coherence properties that cannot be accounted for in geometrical optics [13].

\section{ACKNOWLEDGMENTS}

We acknowledge support from Projects of the Spanish Ministerio de Economía y Competitividad No. MTM201239101-C02-01 (M.A.P.), No. FIS2013-41709-P (M.A.P.), No. FIS2013-41709-P (I.G.), and No. FIS2012-35583 (A. L.).
[1] L. A. Khalfin, Zh. Eksp. Teor. Fiz. 33, 1371 (1957) [Sov. Phys. JETP 6, 1053 (1958)]; C. Teuscher (ed.), Alan Turing: Life and Legacy of a Great Thinker (Springer, Berlin, 2004); A. Degasperis, L. Fonda, and G. C. Ghirardi, Nuovo Cimento A 21, 471 (1974); B. Misra and E. C. G. Sudarshan, J. Math. Phys. 18, 756 (1977).

[2] Maybe Greek philosophy was not the best reference for this effect. This has more to do with the Eastern nonduality solutions of the observer-observed apparent polarity, which recalls the object-apparatus entanglement present in any observation process.

[3] D. Wallace, Phys. Rev. A 63, 022109 (2001).

[4] P. Facchi and S. Pascazio, Phys. Rev. Lett. 89, 080401 (2002); J. Phys. A 41, 493001 (2008).

[5] A. G. Fox and T. Li, Bell Syst. Tech. J. 40, 453 (1961).

[6] L. Ronchi, Appl. Opt. 9, 733 (1970).

[7] M. Moshinsky, Phys. Rev. 88, 625 (1952); E. Torrontegui, J. Muñoz, Y. Ban, and J. G. Muga, Phys. Rev. A 83, 043608 (2011).

[8] J. M. Raimond, C. Sayrin, S. Gleyzes, I. Dotsenko, M. Brune, S. Haroche, P. Facchi, and S. Pascazio, Phys. Rev. Lett. 105, 213601 (2010); J. M. Raimond, P. Facchi, B. Peaudecerf, S. Pascazio, C. Sayrin, I. Dotsenko, S. Gleyzes, M. Brune, and S. Haroche, Phys. Rev. A 86, 032120 (2012); A. Signoles, A.
Facon, D. Grosso, I. Dotsenko, S. Haroche, J.-M. Raimond, M. Brune, and S. Gleyzes, Nat. Phys. 10, 715 (2014); F. Schäfer, I. Herrera, S. Cherukattil, C. Lovecchio, F. S. Cataliotti, F. Caruso, and A. Smerzi, Nat. Commun. 5, 3194 (2014).

[9] M. A. Porras, A. Luis, I. Gonzalo, and A. S. Sanz, Phys. Rev. A 84, 052109 (2011); M. A. Porras, A. Luis, and I. Gonzalo, ibid. 88, 052101 (2013).

[10] G. R. Allcock, Ann. Phys. (N.Y.) 53, 253 (1969); 53, 286 (1969); 53, 311 (1969); I. L. Egusquiza and L. J. Garay, Phys. Rev. A 68, 022104 (2003).

[11] J. G. Muga and C. R. Leavens, Phys. Rep. 338, 353 (2000); J. A. Damborenea, I. L. Egusquiza, G. C. Hegerfeldt, and J. G. Muga, Phys. Rev. A 66, 052104 (2002); G. C. Hegerfeldt, D. Seidel, and J. G. Muga, ibid. 68, 022111 (2003); G. C. Hegerfeldt, J. G. Muga, and J. Muñoz, ibid. 82, 012113 (2010); Time in Quantum Mechanics, Lecture Notes in Physics Vol. 734, edited by J. G. Muga, R. Sala Mayato, and I. L. Egusquiza (Springer-Verlag, Berlin, 2008), Vol. 1; Time in Quantum Mechanics, Lecture Notes in Physics Vol. 789, edited by J. G. Muga, A. Ruschhaupt, and A. del Campo (Springer-Verlag, Berlin, 2009), Vol. 2.

[12] M. Hillery, R. F. O'Connell, M. O. Scully, and E. P. Wigner, Phys. Rep. 106, 121 (1984).

[13] A. Torre, Linear Ray and Wave Optics in Phase Space (Elsevier, Amsterdam, 2005). 